\title{
Vocabulaire de la cité et formes urbaines dans le monde étrusque
}

City's Vocabulary and Urban Forms in the Etruscan World

\section{Claire Joncheray}

\section{(2) OpenEdition}

1 Journals

Édition électronique

URL : https://journals.openedition.org/gaia/1077

DOI : 10.4000/gaia. 1077

ISSN : 2275-4776

Éditeur

UGA Éditions/Université Grenoble Alpes

\section{Édition imprimée}

ISBN : 978-2-37747-199-7

ISSN : 1287-3349

\section{Référence électronique}

Claire Joncheray, "Vocabulaire de la cité et formes urbaines dans le monde étrusque », Gaia [En ligne], 22-23 | 2020, mis en ligne le 30 juin 2020, consulté le 09 décembre 2021. URL : http://

journals.openedition.org/gaia/1077 ; DOI : https://doi.org/10.4000/gaia.1077

Ce document a été généré automatiquement le 9 décembre 2021.

Gaia. Revue interdisciplinaire sur la Grèce archaïque 


\title{
Vocabulaire de la cité et formes urbaines dans le monde étrusque
}

\author{
City's Vocabulary and Urban Forms in the Etruscan World
}

\author{
Claire Joncheray
}

1 Comparer les différents modèles d'utilisation et de représentation du phénomène urbain dans l'Antiquité offre l'occasion de proposer une synthèse concernant le monde étrusque. La spécificité de l'organisation spatiale de cette civilisation entre les $\mathrm{VI}^{\mathrm{e}}$ et $\mathrm{IV}^{\mathrm{e}}$ siècles est nettement apparue depuis la forte implication des recherches dans le domaine de l'archéologie urbaine.

2 Toutefois, l'image et la compréhension par les Étrusques eux-mêmes de leur fonctionnement politique et urbain restent mal connues. Notre maitrise de la langue étrusque, dans l'état actuel de nos connaissances, ne permet pas encore de distinguer avec assurance l'ensemble du vocabulaire lié à la ville, malgré la certitude du lien entre la racine ${ }^{*} s p u(r)$ et un aspect communautaire ${ }^{1}$.

Il s'agit de voir comment l'étude du vocabulaire associé aux lieux publics peut donner une idée de la localisation et de la structuration de ces espaces en ville ${ }^{2}$. Plusieurs sources peuvent aider à avancer dans cette réflexion: d'abord une vue du statut des villes par un regard extérieur, celui des écrivains grecs, faute de textes littéraires étrusques, et d'une littérature latine, quoique très récente par rapport au début du phénomène urbain; ensuite à partir des villes étrusques elles-mêmes et de leur toponymie; enfin grâce à l'identification dans les textes épigraphiques des différents espaces urbains et des charges politiques dont le vocabulaire symbolise l'identité d'une communauté.

4 À partir du point de vue des Grecs, le statut des villes étrusques est associé au mot polis. Il est par conséquent d'autant plus difficile de concevoir la réalité du fonctionnement politique et topographique des poleis étrusques dans la mesure où, dans le monde grec déjà, ce terme est polysémique. Appliqué à une réalité étrangère, le mot polis relève certainement davantage d'une reconnaissance des alliances politiques et économiques ${ }^{3}$ que d'une définition ethnologique et culturelle. Les Grecs reconnaissent des lieux 
urbains qui gèrent un vaste territoire et protègent leur territoire géographique ${ }^{4}$. Ils présentent donc une vision partielle et standardisée d'un monde hellénisé depuis plusieurs siècles; et ils en donnent une image, reflet de leur propre structure culturelle.

Plusieurs textes désignent les Étrusques comme un peuple vivant en cités. Hérodote, le premier, évoque ce thème dans le cadre de l'origine des Étrusques ${ }^{5}$ : les Lydiens, arrivés en Occident, installèrent des cités sur le modèle du système oriental et méditerranéen. À la même période, Thucydide présente les Tyrrhéniens comme un peuple constitué de cités parce que certaines se déclarent d'elles-mêmes prêtes à participer à la guerre contre Syracuse. Pour Strabon, les poleis tyrrhenídes proviennent d'une évolution politique: le Lydien Tyrrhenos fonda douze cités (dōdeka poleis), en plus de celles existant déjà en Campanie. Les cités d'abord regroupées sous une seule bannière se seraient séparées sous la pression des voisins et deviennent indépendantes. Le fait que les Étrusques soient un peuple fondateur de cités devient même un topos littéraire qui les place ainsi parmi les populations civilisées et hellénisées du bassin méditerranéen ${ }^{6}$.

6 Huit villes d'Étrurie sont explicitement nommées poleis dans les textes grecs $\mathrm{du} \mathrm{VI}^{\mathrm{e}}$ au IV ${ }^{e}$ siècle av. J.-C. Il s'agit d'Adria, Capoue, Nola, Norchia, Oinarea, Pise, Populonia et Spina $^{7}$. Les cités d'après les textes grecs correspondent principalement à des emporia ${ }^{8}$ ou des villes côtières dédiées aux échanges (Pise, Adria, Populonia, Spina et, dans une moindre mesure, Capoue et Nola). L'image du monde étrusque proposée par cette liste apparaît disproportionnée au regard des cités connues. En effet, la cité d'Oinarea nous est inconnue, malgré une suggestion d'identification avec Volsinies, et les grandes cités comme Tarquinia, Cerveteri ou Felsina, dont la puissance est confirmée par les découvertes archéologiques, n'ont pas de place dans les fragments littéraires qui nous sont parvenus. Le reflet du système politique et topographique est donc très partiel. Comment comprendre l'attribution du mot polis à des villes qui semblent pour certaines d'entre elles de faible rayonnement? Morgens Herman Hansen, directeur des publications sur le répertoire des cités effectué à partir du grand recensement de l'équipe de Copenhague dans les années 2000 et sur l'évolution du sens à donner au mot polis, propose de définir une polis comme un habitat permanent dont certains éléments topographiques relèvent d'une symbolique propre à la cité ${ }^{\prime}$ : c'est-à-dire une certaine densité urbaine, la séparation des activités commerciales et politiques et la possibilité d'avoir des centres de décision où s'exerce la justice et où s'édictent les lois.

7 La documentation archéologique confirme une partie de la définition de Morgens Herman Hansen, celle d'une polis comme habitat permanent avec une certaine densité urbaine et la séparation des activités commerciales et politiques. Le synœcisme, c'est-àdire le regroupement de villages en ville, se situerait aux alentours du vil ${ }^{e}$ siècle av. J.-C. À partir $\mathrm{du} \mathrm{vl}^{\mathrm{e}}$ siècle, le maillage urbain est donc déjà dense et les habitats sont par conséquent des espaces urbains aménagés, avec des voies de circulation, des remparts, de grands temples en centre-ville et des îlots d'habitation. Marzabotto, née comme une colonie spécialisée dans le commerce et l'artisanat ${ }^{10}$, est l'exemple le plus représentatif et le mieux connu d'une planimétrie élaborée. La cité est fondée au début $\mathrm{du} \mathrm{VI}^{\mathrm{e}}$ siècle av. J.-C. et n'est plus en fonction au $\mathrm{IV}^{\mathrm{e}}$ siècle av. J.-C. Elle possède de grandes voies de circulation qui se croisent à angle droit et une acropole avec cinq édifices religieux et les grands temples dédiés à Tinia et Uni (c'est-à-dire Zeus-Jupiter et Héra-Junon) en centre-ville. Elle est entourée de remparts et de nécropoles hors la ville. Quant aux îlots d'habitations, ils sont divisés en plusieurs maisons à cour centrale de 
dimension très variée. Les cités étrusques apparaissent bien d'un point de vue topographique comme des espaces densément peuplés avec une activité commerciale et résidentielle. Ce type de plan n'est pas la norme ${ }^{11}$ mais il dénote une grande capacité d'adaptation des Étrusques aux recherches topographiques, hygiénistes et scientifiques dans l'air du temps en Méditerranée, surtout en Grèce et en Grande-Grèce.

8 L'aspect topographique de la polis étrusque est bien visible. L'aspect institutionnel présent dans la définition d'une cité à la grecque l'est beaucoup moins. Aucune source archéologique ou littéraire ne permet, dans l'état actuel de nos connaissances, de résoudre la polémique sur la présence d'un corps civique. C'est pourquoi l'expression de Bruno D'Agostino "des cités incomplètes ${ }^{12}$ " reste toujours en suspens. La démonstration de Bruno D'Agostino se fondait sur la définition du peuple dans le monde grec et des sources à disposition pour identifier les traces de ces citoyens ayant la possibilité de s'exprimer par des votes. La question du citoyen-soldat était notamment au cœur de sa réflexion. En effet, le monde grec comme le monde romain est sensible à l'intégration du citoyen dans le corps militaire de manière à générer un cursus honorum, une fraternité entre citoyens, ou encore à responsabiliser le citoyen. Ces données ne sont pas identifiables dans le monde étrusque malgré l'emprunt iconographique fort de l'image des hoplites dans l'art étrusque ${ }^{13}$, car il n'est pas sûr que l'hoplitisme soit lié à un aspect civique comme dans le monde grec.

Un troisième élément est absent de la définition d'une cité : Morgens Herman Hansen avait donné un autre argument pour définir une polis, celui de centres de décision, judiciaire et législatif. Cette donnée n'est pas identifiable. Les places publiques, de type forum ou agora, sont majoritairement ignorées entre les $\mathrm{VI}^{\mathrm{e}}$ et $\mathrm{IV}^{\mathrm{e}}$ siècles, sauf l'unicum de Marzabotto, localisé à l'est du temple de Tinia en centre-ville ${ }^{14}$. Quant aux édifices en forme de théâtre ou d'hémicycle ${ }^{15}$, comme les bouleutéria ou le comitium, symbolisant dans l'Antiquité les lieux de réunion, ils répondent au même problème. Un seul théâtre potentiel, à Cerveteri, dont l'utilisation ne dépasse pas le demi-siècle, a pu être fouillé16. Son unicité rend la compréhension de sa construction et sa chronologie assez difficile à interpréter. La forme urbaine étrusque est assez bien documentée, mais l'usage politique de ces villes reste nettement bien moins connu.

$\mathrm{Si}$ les textes grecs contemporains du phénomène de structuration urbaine des $\mathrm{VI}^{\mathrm{e}}$ et $\mathrm{IV}^{\mathrm{e}}$ siècles avant notre ère utilisent le terme de polis pour désigner les agglomérations étrusques, même si la thématique de l'indépendance de ces cités est récurrente dans ces textes, il n'existe pas de description précise des processus politiques internes aux cités.

11 Le vocabulaire grec montre ses limites à cause de la définition très polysémique du mot polis dont l'ensemble de la sémantique ne regroupe pas la réalité archéologique étrusque. Un autre regard sur l'articulation entre les lieux et les espaces publics est apporté par le vocabulaire étrusque à partir des sources toponymiques.

12 Le nom des cités offre quelques pistes de réflexions sur la manière dont le citadin se sent appartenir à la cité qu'il habite. Le tableau ${ }^{17}$ ci-dessous qui récapitule les différents noms des cités étrusques présente des lacunes : il n'y a pas de données sur le nom étrusque des cités de Pompéi, Prato-Gonfienti, Pise, Adria, Spina et Verucchio. Pour Pontecagnano, Fratte, Pérouse, Cortone ou Chiusi ${ }^{18}$, aucune étude satisfaisante sur l'étymologie des noms de ville n'a été proposée. 
Tableau des ethniques et noms des cités (les mots avec * sont issus d'une reconstitution).

\begin{tabular}{|c|c|c|}
\hline & Nom étrusque & Nom latin \\
\hline Adria & $\varnothing$ & Adria/Atria \\
\hline Arezzo & Aritim(i) & Arretium \\
\hline Bologne & Felsina & $\begin{array}{l}\text { Bononia (après la prise par les } \\
\text { Gaulois) }\end{array}$ \\
\hline Capoue & Velthur*, Capys, Volturnum & Capua \\
\hline Caere & $\begin{array}{l}\text { Cisra, Caisriva*, Ceizra (en grec, } \\
\text { Agylla) }\end{array}$ & Caere \\
\hline Chiusi & Clevsin, Camars & Clusium \\
\hline Cortone & Curtun & Corythus, Cortona \\
\hline Fiesole & Visulis, Vupsul, Vis & Faesulae \\
\hline $\begin{array}{lll}\text { Forcello di Bagnolo } \\
\text { San Vito }\end{array}$ & $\varnothing$ & $\varnothing$ \\
\hline Fratte & Irna* (en grec, Markina) & $\varnothing$ \\
\hline Marzabotto & Kainua & $\varnothing$ \\
\hline Pérouse & $\varnothing$ & Perusia, Perusium, Pirusio \\
\hline Pise & $\varnothing$ & Pisa \\
\hline Pompéi & $\varnothing$ & Pompeii \\
\hline Pontecagnano & Aminaia* & Picentia \\
\hline Populonia & Pupluna, Puplana, Pufluna, Fufluna & Populonium, Populonia \\
\hline Prato-Gonfienti & $\varnothing$ & $\varnothing$ \\
\hline Roselle & $\varnothing$ & Rusellae \\
\hline Spina & $\varnothing$ & Spina \\
\hline Tarquinia & Tarchnalth(i) & Tarquinii \\
\hline Véies & Vei & Veii \\
\hline Verucchio & $\varnothing$ & $\varnothing$ \\
\hline Vetulonia & Vatl, Vetluna, Vetalu, Valtuna & Vetulonia \\
\hline Vezlna - Orvieto & Velzna & Volsinii \\
\hline
\end{tabular}




\begin{tabular}{|l|l|l|}
\hline Volterra & Velathri & Volaterrae \\
\hline Vulci & Velc/Velch & Vulci \\
\hline
\end{tabular}
prophétesse dont le nom a été rapproché de celui d'une figure du mythe grec, Mantô, mère de Ocnus (le fondateur de la ville) et fille de Tirésias ${ }^{19}$, ou le nom de Véies qui proviendrait de la déesse Vei à laquelle un temple serait consacré dans la ville ${ }^{20}$. D'autres cités sont liées à des réalités géographiques comme Capoue qui correspondrait au nom du fleuve Volturnum ${ }^{21}$, ou comme Roselle (Rusellae) qui pourrait aussi avoir un rapport avec la nature du lieu où la cité a été créée, c'est-à-dire les torrents ${ }^{22}$. distingue par le grand nombre de ses appellations et leurs différentes interprétations. En grec, la cité s'appelle Agylla ${ }^{23}$, nom considéré comme pélasgique alors que Caere serait le nom étrusque. D'après Stéphane de Byzance (s.v. Agylla), Agyllio pourrait signifier également «le bronze", compte-tenu de sa production artisanale ou de l'utilisation de ce matériau dans son armement. La version phénicienne, d'après les lamelles de Pyrgi du v viècle, serait Kaisria. En étrusque, le nom de la cité serait Cisra ${ }^{24}$. Sur une inscription de la seconde moitié du Iv ${ }^{e}$ siècle av. J.-C., sur un des sarcophages en marbre de la tombe de la famille des Tamsnie, se lit Caisriva, interprétée comme le nom ethnique de la ville de Caere ${ }^{25}$. communauté. Cet argument pourrait associer les deux aspects, topographique et communautaire. L'expression «ville nouvelle » a été identifiée pour deux cités : Nola et Marzabotto. La cité de Nola, à la fin du v viècle et pendant tout le IV ${ }^{\mathrm{e}}$ siècle, frappe des monnaies avec l'inscription Uri. Giovanni Alessio propose d'y voir le nom le plus ancien de Nola qui serait un dérivé d'une forme osque signifiant « la ville nouvelle ». Pour la cité de Marzabotto ensuite, l'inscription ---]ni kainuathi x[-- désignerait au locatif le nom Kainua. Il s'agirait d'une formule parlante mise en rapport avec le grec kainòs/ kainòn qui signifie «nouveau ». Marzabotto s'appellerait Kainua, ce qui pourrait se traduire par « ville nouvelle » d'après Giuseppe Sassatelli. L'interprétation du nom de Visul (Fiesole) ferait intervenir le terme de spura qui désigne en langue étrusque une ville ou une communauté d'après Nicola Rilli, à partir de la dérivation des expressions pural visulis, vupsul, spural vis ${ }^{26}$. Le nom de Populonia dériverait du terme peuple ${ }^{27}$. La difficulté réside dans la possibilité de faire coïncider deux échelles : l'ethnique des cités et la notion de « public » comprise dans la racine ${ }^{*}$ Rasn ${ }^{28}$.

racine * Rasn- suggère la vision d'un peuple étrusque commun et non une division par cité. Cette division se retrouve au contraire dans la thématique des dodécapoles étrusques. Le pluriel sous-entend que la liste des cités formant la ligue des douze villes varie dans le temps, mais aussi qu'il semble exister plusieurs ligues comme celles de l'Étrurie septentrionale, centrale et méridionale ${ }^{29}$. La création à la période augustéenne du praetor Etruriae se fonde sur l'antériorité de la dodécapole étrusque, mais cet argument a posteriori ne permet pas vraiment d'être sûr de la fonction et de l'organisation de cette ligue pour la période classique. La vision d'un peuple qui soit lié à une cité est assez difficile à préciser, car le statut des villes peut changer. Mario Torelli avait suggéré que l'organisation sociale des cités se fonde sur le système des 
tribus comme pour le monde latin ${ }^{30}$, ce qui ne peut être démontré pour toutes les villes étrusques.

17 Le monde étrusque se compose d'une série de communes dont le cloisonnement politique ne semble pas assuré. Il est possible d'être magistrat d'une cité et d'une autre sur un même cursus honorum ${ }^{31}$, ce qui montre la souplesse du système politique et son grand libéralisme. Par exemple, sur une inscription d'Orvieto, un certain Vel ${ }^{32}$ a exercé plusieurs fonctions que nous ne traduisons pas faute d'équivalence exacte. Il aurait été maru spurana et purt à Volsinies (l'actuelle Orvieto); puis certainement suite à des

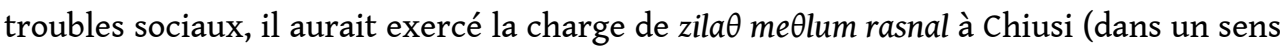
local et non en rapport avec la ligue étrusque); enfin il aurait exercé le pulum à Rome, même si la lecture du mot rumitrinethi pouvant désigner Rome est très discutée. Les noms des magistratures sont souvent associés à des qualificatifs qui en précisent le sens. Il semble que le zilat puisse être une charge suprême de magistrature et le maru le plus bas degré en rapport avec un collège militaire voire éventuellement religieux ${ }^{33}$. Ce sont les deux charges publiques du cursus qui peuvent être associées au mot étrusque désignant la communauté urbaine, c'est-à-dire spu/spura. Giovanni Colonna a suggéré de rapporter cette formule zila $s p u$ à une fonction urbaine comme le praetor urbain latin ou un astynomos grec. Luciana Aigner Foresti constate que la famille Alethna de Musarna, par exemple, possède toujours la magistrature du zila $\theta$ citadin, qui serait éponyme et annuelle : il pourrait s'agir d'un principe dynastique ou d'un équivalent du consul romain. La sphère de compétence de ce zila $\theta$ est peut-être liée aux confins du territoire ou à des villes satellites de la cité principale ${ }^{34}$.

L'identification de ces magistratures relève d'un questionnement qui s'affine de plus en plus avec la recherche par le contexte textuel et iconographique. Toutefois, il est encore difficile de définir ces magistratures en lien avec la communauté urbaine à cause d'un déséquilibre chronologique et géographique des sources. $90 \%$ de la documentation épigraphique sur les magistratures provient de l'Étrurie méridionale et la majorité de la documentation est datable du IV siècle au II siècle av. J.-C. Il se peut que, au $\mathrm{v}^{\mathrm{e}}$ siècle, l'émergence des curricula sur les épitaphes soit le signe de l'affirmation du poids des grandes familles ${ }^{35}$. Le nom étrusque n'est pas encore attesté pour chaque cité, ce qui rend la comparaison un peu difficile.

19 Maintenant que la cité étrusque a été définie comme un espace densément peuplé avec des activités marchandes et artisanales, forte d'un ensemble de magistrats qui traitent des affaires publiques, malgré toutes les limites de nos connaissances, nous pouvons réfléchir sur la manière dont les Étrusques comprenaient le fonctionnement topographique de leur cité et les différents lieux publics. Le travail fondamental de

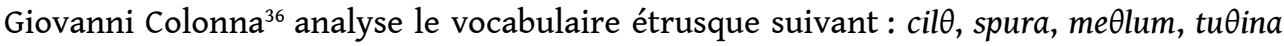
et rasna, qu'il traduit par arx, ciuitas, agglomération, peuple et citoyens en armes. Ces définitions ne font pas l'unanimité, car elles se fondent sur un principe d'analogie avec les mondes grecs, latins et osques, calquant ainsi le vocabulaire sur une même division topographique et communautaire entre ces quatre peuples. Par exemple, tüina pourrait définir aussi une communauté rurale qui n'aurait pas d'autonomie juridique et dépendrait de centres urbains dont elle serait une émanation ${ }^{37}$ et la racine ${ }^{*}$ Rasnpourrait désigner le peuple étrusque ${ }^{38}$. Par conséquent, trois mots de vocabulaire sont plus importants pour notre recherche sur le lien entre espace et lieu public : cilt, spura, me日lum. 

institutionnel référencé dans le texte du Liber linteus ${ }^{39}$ et dans des documents épigraphiques des cités de Volsinies et de Tarquinia. Quant au mot spura, l'occurrence la plus ancienne est incisée sur un objet de Volsinies du viI siècle. Mais la majorité des occurrences des mots de la famille de *spur- concerne les charges du cursus honorum municipal, écrites sur le Liber linteus. Ainsi, les mots de la famille de ${ }^{*} s p u(r)$ appartiennent majoritairement à des expressions rituelles peu traduisibles. Il est ainsi difficile d'estimer la part de l'élément citadin dans ces formules consacrées. Différentes définitions ont été proposées pour le mot spura. Elles sont répertoriées dans un article de Giovanni Alessio: on entend par spura, città, confine, estero, società, popolo et territorio ${ }^{40}$. Pour Giovanni Colonna, dont l'étude est plus convaincante, spura serait l'équivalent de communautés organisées au sens le plus ample sans distinction de genre, d'âge ou d'état social et correspondrait à la notion de ciuitas ${ }^{41}$. L'aspect topographique de spura peut se matérialiser dans les formules liées au bornage ${ }^{42}$.

21 Les mots MeOlum et spura sont, tous les deux, en rapport avec les confins et ils contiendraient, tous les deux, des notions topographiques. On lit me日lum sur une borne du territoire à Bolsena : le cippe de Bolsena avec l'inscription $\theta v a l$ me日lumes (ET Vs 8.3) a été retrouvé devant une des entrées basses de la ville. Elle serait en relation avec le pomerium urbain. Le cippe parle des confins de la ville et non pas de l'ager quel que soit le sens du premier $\operatorname{mot}^{43}$. Me日lum serait une entité topographique qui correspondrait à l'urbs, alors que le spura serait un équivalent de la ciuitas et associerait la cité et son ager. Le bornage est en effet une affaire de topographie plutôt que de citoyens. Toutefois, en règle générale, l'utilisation du terme tular est essentiellement en rapport avec le mot spura et non pas me日lum, mais l'expression des limites de la cité peut employer les deux termes, sans qu'une distinction soit compréhensible dans l'état actuel de nos connaissances. Pour la majorité des commentateurs, spura ne correspond pas à une entité topographique d'autant plus que les expressions du bornage sont essentiellement liées à la cité de Fiesole. Il pourrait s'agir d'un épiphénomène.

Parmi les mots institutionnels, un seul désignerait une partie topographique de la ville. Il s'agit du mot cil , interprété comme pouvant désigner l'acropole ou un temple sur l'acropole à identifier comme l'édifice que les Latins appellent arx. C'est en comparant le système étrusque à ses équivalents dans les mondes grec, romain et italique que l'espace situé dans la partie haute de la ville a été associé au mot étrusque cill. Ce mot apparaît uniquement dans le Liber linteus. Les formules de ce texte de référence sont toutefois figées et reflètent des rites dont le contexte et la signification nous échappent. Du fait de l'association du terme cilo avec sacnica (lieu sacré) et de sa déclinaison au locatif, Giovanni Colonna donne à ce terme un sens topographique et religieux. Il s'agirait au moins d'un sanctuaire en lien avec le cadre général de la cité.

Aldo Luigi Prosdocimi était allé plus loin dans la signification de ce mot et proposait une association avec l'idée de l'arx ${ }^{44}$. En effet, dans le Liber linteus, trois espaces

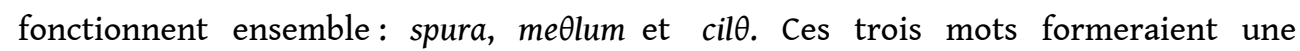
tripartition qui correspondrait au grec astu-polis-acropole ; au latin urbs-ciuitas-arx; et chez les Ombriens au rapport entre touta et ocar dans les textes épigraphiques des tables eugubines (le premier désignant la communauté, le second un lieu topographique sacré). En comparant ces trois situations différentes, Mauro Cristofani propose également d'associer, par élimination, le mot cill à l'arx puisque le mot spura désignerait la communauté et le mot me日lum correspondrait à la ville.

Gaia, 22-23 | 2020 

l'unanimité chez les linguistes, a été rapportée par Dieter Steinbauer et Jean HadasLebe $^{45}$. Il s'agirait de comprendre dans le mot cil $\theta$ l'association avec le mot $c i$ qui veut dire le chiffre trois. Ainsi Dieter Steinbauer associe le mot cil à un aspect tripartite. Dans le Liber Linteus, cil $\theta$ apparait essentiellement dans deux formules plusieurs fois répétées, l'une à l'ablatif, l'autre au locatif-instrumental. Jean Hadas-Lebel propose que les Étrusques, tout en vivant dans des cités-États bien distinctes (les spura), étaient conscients d'appartenir à un même peuple et qu'ils avaient créé une ligue rassemblant les principales de leurs métropoles et se réunissant chaque année à Volsinies, près du temple de Voltumna ${ }^{46}$. Nous ignorons le nom que les Étrusques eux-mêmes donnaient à leur confédération (peut-être était-ce précisément me日lum). Quoi qu'il en soit, il est possible que le mot étrusque cil $\theta$ ait servi à désigner une subdivision non pas à l'intérieur de la cité (spura), mais à l'intérieur de la nation étrusque (me日lum ?). Autrement dit, chaque spura indépendante était en même temps cil $\theta$ au sein du plus vaste ensemble que constituait le nomen etruscum. Ce qui signifierait que la spura et la cil $\theta$ mentionnées dans le Liber Linteus entraient dans un rapport de coréférence. Pour Jean Hadas-Lebel, le mot cil $\theta$ désignerait une subdivision à l'intérieur d'un ensemble ethnique plus vaste. même temps à une identification sociale et politique dans le monde grec. Ce modèle a été utilisé dans plusieurs études pour essayer de comprendre le vocabulaire étrusque et appliquer cette polysémie également au monde étrusque. Toutefois, même si les Étrusques possèdent un système urbain dès la fin de la période archaïque, la visibilité $\mathrm{du}$ fonctionnement municipal et sa mise en scène dans la glorification posthume des individus se multiplient à partir $\mathrm{du} \mathrm{IV}^{\mathrm{e}}$ siècle sous la pression politique, militaire et culturelle de Rome. Si on étudie l'organisation planimétrique des cités, il existe plusieurs éléments constitutifs de la ville d'un point de vue topographie : l'acropole, le grand temple et les murailles. Pour le moment, aucun mot de vocabulaire n'est le reflet sémantique de ces unités qui construisent l'identité d'une cité.

Entre les $\mathrm{VI}^{\mathrm{e}}$ et $\mathrm{IV}^{\mathrm{e}}$ siècles, il semble que le monde étrusque puisse être compris comme un monde avec de forts liens aristocratiques où on peut être membre de plusieurs communautés politiques. Le cloisonnement ne semble pas aussi fermé que l'image diffusée par le monde grec. Le fonctionnement civique peut relever d'un attachement à plusieurs familles plutôt que d'une relation à un espace politique unique ou à une ville précise. Si tout le territoire n'est pas forcément occupé, les centres urbains ne sont pas le lieu exclusif du pouvoir dans un monde de migration et d'installations sur plusieurs terres.

La reconnaissance par le vocabulaire étrusque des espaces spécifiques de la ville est un bon exemple d'étude en cours et ouvre encore de belles perspectives de recherche. Notamment sur la manière dont les Étrusques vivent leur sentiment d'appartenance à une cité. 


\section{BIBLIOGRAPHIE}

\section{Abréviations}

- BTCGI = NENCI Giuseppe \& VALLET Georges (dir.), Bibliografia topografica della colonizzazione greca in Italia e nelle Isole Tirreniche, Pise/Rome/Naples, Scuola normale superiore, École française de Rome, Centre J. Bérard, 1977-2012.

- ET = RIX Helmut (dir.), Etruskische Texte. Editio minor, Tübingen, G. Narr, 1991.

- FGrHist = JACOBY Felix, Die Fragmente der griechischen Historiker, Berlin, Weidmann, Leyde/New York/ Cologne, E. J. Brill, 1923-1999.

- FHG = MÜLLER Karl O., MÜLLER Theodor \& LETRONNE Antoine J., Fragmenta Historicorum Graecorum, Paris, Firmin Didot, 1841.

- GGM = MÜLLER Karl, Geographi Graeci minores, Paris, Firmin Didot, 1855.

ADAM Anne-Marie \& ROUVERET Agnès, « Les cités étrusques et la guerre au v siècle av. notre ère ", dans Crise et transformation des sociétés archaïques de l'Italie antique au ve siècle av. J.-C. (Actes de la table ronde de Rome, 19-21 novembre 1987), Rome, École française de Rome, 1990, p. 327-356.

AIGNER FORESTI Luciana, « La lega etrusca », dans L. Aigner Foresti, A. Barzanò, C. Bearzot \& L. Prandi (éd.), Federazioni e federalismo nell'Europa antica alle radici della casa comune europea, vol. I, Milan, Vita e Pensiero, 1994, p. 327-350.

ALESSIO Giovanni, « Osservazioni sui riflessi latini e sabini dell'Etr. spur- "città" », dans G. A. Mansuelli \& R. Zanchieri (éd.), La città etrusca e italica pre-romana, Bologne, Istituto per la storia di Bologna, 1970, p. 361-364.

BELFIORE Valentina, Il Liber linteus di Zagabria. Testualità e contenuto, Pise/Rome, Fabrizio Serra, 2010.

BOURDIN Stéphane, Les peuples de l'Italie préromaine : identités, territoires et relations inter-ethniques en Italie centrale et septentrionale (VIII ${ }^{e} \mathrm{I}^{\text {er }}$ s. av. J.-C.), Rome, École française de Rome, 2012.

BRACCESI Lorenzo, « Ierone, Erodoto e l'origine degli Etruschi », Hesperia, 9, 1998, p. 53-62. BRESSON Alain, L'économie de la Grèce des cités (fin $\mathrm{VI}^{e}-\mathrm{I}^{e r}$ siècle a. C.), vol. I, Paris, A. Colin, 2007. BRIQUEL Dominique, Les Pélasges en Italie : recherches sur l'histoire de la légende, Rome, École française de Rome, 1984.

BRIQUEL Dominique, «Le regard des Grecs sur l'Italie indigène », dans Crise et transformation des sociétés archaïques de l'Italie antique au ve siècle av.J.-C. (Actes de la table ronde de Rome, 19-21 novembre 1987), École française de Rome, 1990, p. 165-188.

CERCHIAI Luca, I Campani, Milan, Longanesi, 1995.

COLONNA Giovanni, « Intervento », Dibattito, AION, VI, 1984, p. 257-259.

COLONNA Giovanni, « Il lessico istituzionale etrusco e la formazione della città (specialmente in Emilia Romagna) », dans G. Bermond Montanari (éd.), La formazione della città in Emilia-Romagna. Prime esperienze urbane attraverso le nuove scoperte archeologiche, Imola, Istituto per la Storia di Bologna, 1987, p. 15-24.

COLONNA Giovanni, « Strutture teatriformi in Etruria », dans J.-P. Thuillier (éd.), Spectacles sportifs et scéniques dans le monde étrusco-italique, Rome, École française de Rome, 1993, p. 321-347. 
COMELLA Annamaria \& STEFANI Grete, Materiali votivi del santuario di Campetti a Veio, scavi 1947 e 1969, Rome, Giorgio Bretschneider éd., 1990.

CRISTOFANI Mauro, « L'edificio ellittico », dans M. Cristofani, V. Bellelli et al. (éd.), Caere, vol. 4 : Vigna Parrocchiale: scavi 1983-1989, Rome, Consiglio Nazionale delle Ricerche, 2003, p. 249-254.

D'AGOSTINO Bruno, « Military Organization and Social Structure in Archaic Etruria », dans

O. Murray \& S. Price (éd.), The Greek City from Homer to Alexander, Oxford, Clarendon Press, 1990, p. 59-83.

DE CARO Stefano et al., « La Campania. L'attività della Soprintendenza Archeologica di Napoli e Caserta nel 1996 », dans Mito e storia in Magna Grecia (Atti del XXXVI Convegno di studi, Taranto, 4-7 ottobre 1996), Tarente, Istituto per la storia e l'archeologia della Magna Grecia, 1997, p. $401-452$.

DELLA FINA Giuseppe Maria (éd.), I Greci in Etruria, Rome, Edizioni Quasar, 2004.

DE SIMONE Carlo, «L'aspetto linguistico », dans C. M. Stibbe, G. Colonna \& C. De Simone (éd.), Lapis Satricanus: Archaeological, Epigraphical, Linguistic and Historical Aspects of the New Inscription from Satricum, La Haye, Staatsuitgeverij, 1980, p. 71-94.

DE SIMONE Carlo, «Volsinii e i duodecim populi nella documentazione epigrafica », dans Volsinii $e$ la dodecapoli etrusca (Annali della Fondazione per il Museo «Claudio Faina», 2), Orvieto, La Fondazione, 1985, p. 89-100.

DEVOTO Giacomo, « Nomi di divinità etrusche », Studi Etruschi, 6, 1932, p. 243-260.

FIRPO Giulio, « Quale Sentinum? », dans M. Medri (éd.), Sentinum 295 a.C. Sassoferrato 2006, 2300 anni dopo la battaglia. Una città romana tra storia e archeologia, Rome, «L'Erma » di Bretschneider, 2008, p. 91-97.

GOVI Elisabeta (éd.), Marzabotto una città etrusca, Bologne, Ante Quem, 2007.

HADAS-LEBEL Jean, « Le nom de la tribu en latin, en ombrien et en étrusque », De lingua latina. Revue de linguistique latine du Centre Alfred Ernout, 9, 2016, p. 1-14.

HADAS-LEBEL Jean, « Essere greco in Etruria », dans L. Aigner-Foresti \& P. Amann (éd.), Beiträge zur Sozilageschichte der Etrusker (Akten der internationalen Tagung, Wien, 8.-10. Juni 2016), Holzhausen Verlag, coll. « PHERSU. Etrusko-italische Studien, vol. 1 », 2018, p. 371-381.

HANSEN Morgens Herman, «Introduction », dans M. H. Hansen (éd.), A Comparative Study of Thirty City-State Cultures, an Investigation Conducted by the Copenhagen Polis Centre, Copenhague, C. A. Reitzels Forlag, 2000, p. 11-34.

HANSEN Morgens Herman, « Introduction », dans M. H. Hansen \& E. Nielsen (éd.), A Comparative Study of Six City-State Cultures, an Investigation Conducted by the Copenhagen Polis Centre, Copenhagen, C. A. Reitzels Forlag, 2002, p. 7-22.

HEURGON Jacques, « Les tribunes des spectateurs dans les peintures étrusques », Bulletin de la Société nationale des antiquaires de France, 1961, p. 179-183.

JANNOT Jean-René, « Les cités étrusques et la guerre. Remarques sur la fonction militaire dans la cité étrusque », Ktéma, 10, 1985, p. 127-142.

JONCHERAY Claire, « La ville étrusque classique : du modèle urbain à la réalité multiple et méconnue », dans D. Gracia (éd.), L'habitat en Europe celtique et en Méditerranée préclassique. Domaines urbains, Paris, Errance, 2013a, p. 139-151. 
JONCHERAY Claire, «Les invisibles du processus de décision dans les cités étrusques à la période classique et leurs enjeux historiographiques ", dans A. Choné \& P. Hamman (éd.), Villes et démocratie, Paris, Éditions Orizons, 2013b, p. 63-83.

LAMBRECHTS Roger, Essai sur les magistratures des Républiques étrusques, Bruxelles, Palais des Académies, 1959.

LAMBRECHTS Roger, Les inscriptions avec le mot «tular » et le bornage étrusques, Florence, L. S. Olschki éd., coll. « Biblioteca di Studi Etruschi, 4 », 1970.

LIPPOLIS Enzo, « Nuovi dati sull'acropoli e sulla forma urbana », dans E. Govi \& G. Sassatelli (éd.), Culti, forma urbana e artigianato a Marzabotto. Nuove prospettive di ricerca (Atti del Convegno di studi, Bologna, 3-4 giugno 2003), Bologne, Ante Quem, 2005, p. 139-165.

LUBTCHANSKY Natacha, Le cavalier tyrrhénien : représentations équestres dans l'Italie archaïque, Paris, De Boccard, 2005.

MAETZKE Guglielmo, « Il santuario etrusco italico di Castelsecco (Arezzo) », Rendiconti della Pontificia Accademia Romana di Archeologia, 55-56, 1982-1984, p. 35-53.

MAGGIANI Adriano, « N 100 Rivista di Epigrafia Etrusca », Studi Etruschi, 46, 1978, p. 358-359.

MAGGIANI Adriano, « Appunti sulle magistrature etrusche », Studi Etruschi, 62, 1996, p. 63-94.

MAGGIANI Adriano, « Magistrature cittadine, magistrature federali », dans La lega etrusca dalla dodecapoli ai quindecim populi (Atti della Giornata di studi, Chiusi, 9 ottobre 1999), Pise/Rome, Istituti Editoriali Poligrafici Internazionali, 2001, p. 38-49.

MAGGIANI Adriano, « La libbra etrusca. Sistemi ponderali e monetazione », Studi Etruschi, 65-68, 2002, p. 163-199.

MASSA-PAIRAULT Françoise-Hélène, La cité des Étrusques, Paris, CNRS Éditions, 1996.

MAZZOLAI Aldo, Roselle e il suo territorio, Grossete, Tipografia Stem, 1960.

MELE Alfonso, Il commercio arcaico, prexis et emporie, Naples, Centre Jean Bérard, 1979.

MINTO Antonio, Populonia, Florence, Istituto di Studi Etruschi, 1943.

MORANDI TARABELLA Massimo, Prosopografia etrusca, vol. I : Corpus, 1. Etruria meridionale, Rome, « L'Erma » di Bretschneider, 2004.

PAQUOT Thierry, L'espace public, Paris, La découverte, 2010.

PROIETTI Giuseppe, «L'ipogeo monumentale dei Tamsnies, considerazioni sul nome etrusco di Caere e sulla magistratura cerite nel IV sec. a.C. », Studi etruschi, 51, 1985, p. 570-571.

PROSDOCIMI Aldo Luigi, « Il lessico istituzionale italico. Tra linguistica e storia », dans La Cultura italica (Atti del Convegno della Società Italiana di Glottologia, Pisa, 19-20 dicembre 1977), Pise, Giardini éd., 1978, p. 29-74.

RILLI Nicola, Gli Etruschi a Sesto Fiorentino, Florence, Tipogra fiagiuntina, 1964.

RIX Helmut, « Etrusco mechl rasnal = lat. res publica », dans M. G. Marzi Costagli \& L. Tamagno Perna (éd.), Studi di antichità in onore di Guglielmo Maetzke, Rome, G. Bretschneider éd., 1984, p. $454-468$.

SASSATELLI Giuseppe, « Un altro documento epigrafico e il nome etrusco della città », dans E. Govi \& G. Sassatelli (éd.), Culti, forma urbana e artigianato a Marzabotto. Nuove prospettive di ricerca (Atti del Convegno di studi, Bologna, 3-4 giugno 2003), Bologne, Ante Quem, 2005, p. 47-55. 
SASSATELLI Giuseppe, « Il tempio di Tina a Marzabotto e i culti della città etrusca », dans G. Cresci Marrone \& M. Tirelli (éd.), Altinoi, il santuario altinate: strutture del sacro a confronto e i luoghi di culto lungo la via Annia (Atti del Convegno, Venezia, 4-6 dicembre 2006), Rome, Edizioni Quasar, 2009, p. 325-344.

STEINBAUER Dieter, Neues Handbuch des Etruskischen, St. Katharinen, Scripta Mercaturae Verlag, 1999.

TAGLIONI Caterina, «L'abitato », dans G. Sassatelli \& C. Morigi Govi (éd.), Da Felsina a Bononia, dalle origini al XII secolo, Bologne, Grafis, 1996, p. 17-30.

THUILLIER Jean-Paul, Les jeux athlétiques dans la civilisation étrusque, Rome, École française de Rome, 1985.

TORELLI Mario, « Veio, la città, l'arx e il culto di Giunone Regina », dans H. Blanck \& S. Steingräber (éd.), Miscellanea Archaeologica Tobias Dohrn dedicata, Rome, Giorgio Bretschneider éd., 1982, p. 117-128.

TORELLI Mario, «The Etruscan City-State », dans M. H. Hansen (éd.), A Comparative Study of Thirty City-State Cultures, Copenhague, C. A. Reitzels Forlag, 2000, p. 189-208.

VAGNETTI Lucia, Il deposito votivo di Campetti a Veio, Florence, Sansoni, 1971.

VAN DER MEER Lammert Bouke, Liber Linteus Zagrabiensis. The Linen Book of Zagreb: A Comment on the Longest Etruscan Text, Louvain, Peeters éd., 2007.

VAN HEEMS Gilles, « Idéologie et écriture : réflexions sur les mentions de titres et magistratures dans les inscriptions étrusques », dans M.-L. Haack (éd.), L'écriture et l'espace de la mort (Actes du colloque international, Rome, 5-7 mars 2009), Rome, École française de Rome, 2015. Disponible en ligne sur <https://books.openedition.org/efr/2733>.

WATMOUGH Margaret M. T., Studies in the Etruscan Loanwords in Latin, Florence, L. S. Olschki, coll. «Biblioteca di Studi Etruschi, 33 », 1997.

\section{NOTES}

1. Bourdin (2012, 224-239).

2. Pour une définition de l'espace public, voir Paquot (2010). Thierry Paquot présente le sens premier du concept d'espace public comme étant un lieu de discussion.

3. Briquel (1990).

4. Sur la présence des Grecs en Étrurie, voir Della Fina (2004) et Hadas-Lebel (2018).

5. Hérodote, Histoires, I, 94 ; Braccesi (1998).

6. Thucydide, La guerre du Péloponnèse, VI, 88, 6 ; Strabon, Géographie, V, 4, 3.

7. Pour Nola et Capoue, cf. Stéphane de Byzance, s. v. Kó $\pi v \alpha$ et N $\tilde{\omega} \lambda \alpha$ (= Hécatée, FGrHist, IA, 1 , F61; FHG, I, 2, 28 et FGrHist, IA, 1, F62 ; FHG, I, 2, 27) ; pour Adria, cf. Stéphane de Byzance, s. v. Ad pía (= Hécatée, FGrHist, IA, 1, F90 ; FHG, I, 4, 58), Strabon, Géographie, VII, 5, 9 (= Théopompe, FGrHist, IIB, 115, F129 ; FHG, I, 302, F140), Etymologicum Magnum (= Eudoxe, Histoires, IX ; FGrHist, IIA, 79, F1 ; FHG, IV, 407, 2) ; pour Norchia, cf. Philistos (Stéphane de Byzance, s. v. Noukpí $\alpha=$ FGrHist, IIIB, 556, F43; FHG, I, 189, 41); pour Oinarea, cf. Pseudo-Aristote, De mirabilibus auscultationibus, $839 \mathrm{a}-\mathrm{b}$; pour Populonia, cf. Timée (Diodore, Bibliothèque historique, V, 9 = FGrHist, IIIB, 566, F164) ; pour Spina, cf. Hellanicos (Denys d'Halicarnasse, Antiquités romaines, I, $28=$ FGrHist, IA, 4, F4 ; FHG, I, 45, 1) et Scylax (Périple, 17, GGM, I, 25, 17) ; pour Pise, cf. Scylax (Périple, 17, GGM, I, 25, 17). Certains auteurs confondent les villes de Crotone et Cortone; Denys d'Halicarnasse (Antiquités romaines, I, 28) place la ville de Crotone dans le monde des Ombriens, 
alors que l'établissement du texte d'Hérodote (Histoires, I, 57) dans la tradition directe suggère la ville de Kretôn en Chalcidique. Le terme polis est associé aussi à cette ville tyrrhénienne de l'intérieur des terres chez Hérodote (Histoires, I, 56-57) et Hellanicos (Denys d'Halicarnasse, Antiquités romaines, I, 28 = FGrHist, IA, 4, F4 ; FHG , I, 45, 1). Voir aussi Briquel (1984).

8. Les emporia sont des installations commerciales accessibles à l'issue d'un trajet maritime. La notion de commerce l'emporte au v $\mathrm{v}^{\mathrm{e}}$ siècle. Ce sont des espaces propices aux contacts où vit une population mêlée dont les sanctuaires avec leurs divinités protectrices sont le reflet. La place des étrangers y est particulière. Sur le rôle des emporia dans le commerce, voir Mele (1979) et Bresson (2007).

9. La présentation des poleis étrusques a été réalisée par Mario Torelli dans la réflexion plus globale sur la définition du mot grec polis. Voir Torelli (2000) et Hansen (2000, 2002, 2004).

10. Plusieurs maisons cumulent une fonction résidentielle et une fonction artisanale surtout dans le domaine des métaux et des céramiques. Voir Govi (2007).

11. Joncheray (2013a).

12. D'Agostino (1990).

13. La cavalerie semble plus importante que l'infanterie. Sur l'art de la guerre en Étrurie, voir Adam \& Rouveret (1990), Jannot (1985), Lubtchansky (2005).

14. Sur la place publique et le temple de Tinia, voir Lippolis (2005) et Sassatelli (2009). Sur les structures urbaines étrusques, voir Joncheray (2013b).

15. Colonna (1993).

16. À Cerveteri, cf. Cristofani (2003). À Acerra, en Campanie, une seule structure de la fin du $\mathrm{VI}^{\mathrm{e}}$ siècle, ou du début $d u v^{e}$ siècle, est identifiable : sous des sépultures d'époque hellénistique, il s'agit d'un espace circulaire entouré d'un fossé. Son intérieur comporte une série de banquettes : voir De Caro et al. (1997). G. Maetzke signale la présence d'un théâtre en pierre à Castelsecco près d'Arezzo mais de datation plus récente, du $\mathrm{II}^{\mathrm{e}}$ siècle : Maetzke (1982-1985). Sur la structure des bâtiments liés aux jeux, voir Heurgon (1961) et Thuillier (1985).

17. Pour les toponymes recensés, voir BTCGI. À compléter avec: pour Pontecagnano, Colonna (1984) ; pour Fratte, Strabon (Géographie, V, 4, 13) et Cerchiai (1995) ; pour Capoue, noter que Volturnum est considéré comme l'ancien nom étrusque, devenu Capua au moment de la prise par les Campaniens - le locatif Capue apparaît dans l'inscription ET Ta 1.107; pour Véiès, Comella-Stefani (1990); pour Caere, Proietti (1985) - à noter que la forme Ceizra est peut-être un gentilice et la forme Cisra est connue uniquement par Servius (Commentaire à l'Énéide, X, 183); pour Tarquinia, cf. ET Ta 1.17 et AT 1.100 ; pour Arezzo, Steinbauer (1999), ET OB 3.2, et ET Ta 1.263 ; pour Chiusi, le nom Camars est connu seulement par Tite-Live (Histoires romaines, X, 25, 11), cf. Firpo (2008) ; pour Cortone, la forme curtun provient de l'inscription ET Co 4.6 ; Pour Vulci, les légendes étrusques THEZI et THEZLZ apparaissent sur les monnaies : cf. Maggiani (2002) ; pour Roselle, cf. Ptolémée (Géographie, III, I, 43) et l'ethnique Rousilanoi pour Denys d'Halicarnasse (Antiquités romaines, III, 51) et Mazzolai (1960) ; pour Fiesole, cf. Rilli (1964) ; pour Marzabotto, cf. Sassatelli $(2005,52)$.

18. Giovanni Colonna remarque que les noms des cités apparaissent toujours au locatif en rapport avec trois types de noms : une magistrature particulière, une divinité particulière, enfin un produit artisanal spécifique. Une équivalence peut exister entre des gentilices et des noms de cités. Le nom de Tarquin, roi de Rome, aurait été donné à cause de son origine de Tarquinia. Il s'agit cependant du terme latin qui désigne cette cité aussi sous sa forme féminine. Pour Giovanni Colonna, Tarquinia correspond à une magistrature inscrite dans le cursus honorum de Laris Pulenas : cf. Colonna (1984).

19. Apollodore, Bibliothèque, III, 7, 7 ; Diodore de Sicile, Bibliothèque historique, IV, 76 ; ET Co 3.7.

20. Vagnetti (1971) et Maggiani (1978). 
21. Tite-Live, Histoire romaine, IV, 37. On a évoqué aussi le nom du faucon en étrusque, ou encore l'idée d'un héros troyen. Capoue et Volturnum sont les deux noms successifs de la même cité.

22. Aldo Mazzolai suggère un lien avec les sources et les torrents : cf. Mazzolai (1960).

23. Hérodote, Histoires, I, 167; Diodore de Sicile, Bibliothèque historique, XV,14; Denys d'Halicarnasse, Antiquités romaines, I, 20.

24. Servius, Commentaire à l'Énéide, $\mathrm{X}, 183$.

25. Proietti (1985).

26. Alessio (1970) ; pour Marzabotto, cf. Sassatelli $(2005,52)$ et Rilli (1964).

27. Minto (1943) et Watmough (1997). Le terme latin populus aurait une origine étrusque d'après Devoto (1932) et De Simone (1980).

28. De Simone (1985) et Rix (1984).

29. Pour une bibliographie ajournée sur la dodécapole étrusque, voir Bourdin (2012, 299-321).

30. Massa-Pairault (1996) et Torelli (1982).

31. Dans le cursus honorum, la progression serait la suivante : maru, puis cepen, camthi, eisnevc, purt ou macstrevs, et zila $\theta$, d'après Maggiani (2001).

32. ET Vs 1.179. Maggiani (1996) et sur la difficulté d'établissement du texte et du nom de famille du défunt, voir Morandi Tarabella (2004, 281-282).

33. Lambrechts (1959).

34. Aigner Foresti (1994), Colonna (1987) et Maggiani (2001).

35. Pour la variété des formulaires et ses influences, voir Van Heems (2015).

36. Colonna (1987).

37. Taglioni (1996).

38. Le nom Rasenna et la charge de zilath mechl rasnal mériteraient une plus ample discussion.

39. Sur le Liber linteus, voir Belfiore (2010) et Van der Meer (2007).

40. Alessio (1970).

41. Colonna (1987).

42. Lambrechts (1970).

43. Ibid.

44. Prosdocimi (1978).

45. Steinbauer (1999) et Hadas-Lebel (2016).

46. Tite-Live, De urbe condita, $4,23$.

\section{RÉSUMÉS}

Les cités étrusques sont mentionnées dans les textes grecs comme des poleis et les Anciens leur attribuent des caractéristiques helléniques. Certains peuples sont mentionnés ainsi que des héros fondateurs. Le fonctionnement autonome et indépendant des cités se reflète dans leur choix d'alliances ou la présence d'un trésor comme à Delphes. Toutefois il a bien été démontré que ces poleis fonctionnent sur un système politique différent des mondes grec et latin. Les faibles données archéologiques sur les lieux de réunion, l'absence de données littéraires ou épigraphiques sur les citoyens, les différentes sources présentant une armée de mercenaires et non de citoyens-soldats ont fait dire à B. D'Agostino que les cités étrusques étaient des poleis incomplètes. Le vocabulaire étrusque permet cependant d'affiner la notion d'espace public dans

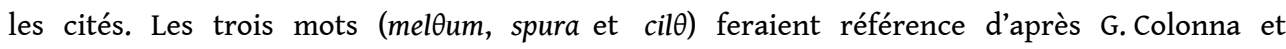


A. Prosdocimi à la morphologie urbaine des cités et à l'espace public. Ces notions correspondraient à une partition comme astu-polis-acropole dans le monde grec ou urbs-ciuitasarx dans le monde latin. La question du rôle de l'arx-acropole-cile, essentielle dans la morphologie urbaine des villes étrusques, renforce la conception religieuse du monde urbain étrusque et pose la question de la place de ce lieu dans la vie politique de ces cités.

The Etruscan cities are mentioned in Greek texts as poleis and the Ancients attribute Hellenic characteristics to them. Certain peoples are mentioned as well as founding heroes. The autonomous and independent organization of the cities is reflected in their choice of alliances or the presence of a treasure as in Delphi. Nevertheless, it has been shown that these poleis operate on a political system different from the Greek and Latin ones. The rarity of archaeological data on the meeting places, the absence of literary or epigraphic data on the citizens, the various sources presenting an army of mercenaries instead of citizen-soldiers lead B. D'Agostino say that the Etruscan cities were incomplete poleis. Etruscan vocabulary, however, makes it possible to

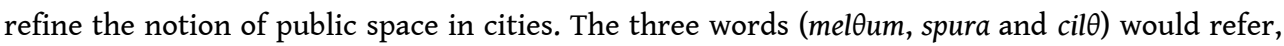
according to G. Colonna and A. Prosdocimi, to the urban morphology of cities and to the public space. These notions would correspond to a partition like astu-polis-acropolis in the Greek world or urbs-ciuitas-arx in the Latin world. The issue of the role of the arx-acropolis-cile, essential in the urban morphology of Etruscan cities, reinforces the religious conception of the Etruscan urban world and raises the question of the function of this place in the political life of these cities.

\section{INDEX}

Mots-clés : cités, urbanisme, monde étrusque, toponymie, organisation politique

Keywords : cities, urbanism, Etruscan civilisation, place names, political organisation

\section{AUTEUR}

\section{CLAIRE JONCHERAY}

Université Paris Nanterre

claire.joncheray@free.fr 